\title{
Potentials of Cellular Vortex Element Modeling of Fluid Flow in Confined 2D Aquifer
}

\author{
Oyetunde Adeoye Adeaga ${ }^{1}$ and Ademola Adebukola Dare ${ }^{2}$ \\ 1. Department of Mechanical Engineering, The Ibarapa Polytechnic, Eruwa 201101, Nigeria \\ 2. Department of Mechanical Engineering, University of Ibadan, Ibadan 200106, Nigeria
}

Received: August 26, 2015 / Accepted: September 25, 2015 / Published: March 31, 2016.

\begin{abstract}
Numerical methods such as finite difference, finite volume, finite element or hybrid methods have been globally used to successfully study fluid flow in porous stratum of which aquifers are typical examples. Those methods involve mathematical expressions which increases computation time with requirement of specific human expertise. In this paper, numerical models for single phase flow in $1 \mathrm{D}$ and 2D using the conservation of mass principles, Darcy's flow equation, equation of state, continuity equation and the STB/CFB (stock tank barrel/cubic feet barrel) balance were developed. The models were then recast into pressure vorticity equations using convectional algorithms. Derived equations were used to formulate transport equations which resemble the conventional vorticity transport equation. Formulated numerical models were used to investigate the daily instantaneous aquifer pressure drawdowns and pressure heads for 365 days. The developed equations were subsequently solved using cellular vortex element technique. The developed computer program was used to investigate confined aquifer of dimensions $10 \times 10 \times 75 \mathrm{~m}$ with single vertex image. For the aquifer rate of $0.5 \mathrm{~m}^{3} / \mathrm{s}, 0.1 \mathrm{~m}^{3} / \mathrm{s}, 0.15 \mathrm{~m}^{3} / \mathrm{s}, 0.2 \mathrm{~m}^{3} / \mathrm{s}, 0.25 \mathrm{~m}^{3} / \mathrm{s}, 1.0 \mathrm{~m}^{3} / \mathrm{s}, 2.0 \mathrm{~m}^{3} / \mathrm{s}, 2.5 \mathrm{~m}^{3} / \mathrm{s}, 3.0 \mathrm{~m}^{3} / \mathrm{s}$, $4.0 \mathrm{~m}^{3} / \mathrm{s}$, the respective average head drawdowns and heads were, $1.127 \pm 0.0141 \mathrm{~m}, 1.317 \pm 0.0104 \mathrm{~m}, 1.412 \pm 0.0041 \mathrm{~m}, 1.427 \pm$ $0.116 \mathrm{~m}, 1.527 \pm 0.0141 \mathrm{~m}, 2.107 \pm 0.0171 \mathrm{~m}, 2.197 \pm 0.0191 \mathrm{~m}, 3.007 \pm 0.0171 \mathrm{~m}, 3.127 \pm 0.0041 \mathrm{~m}, 3.626 \pm 0.0121 \mathrm{~m}$, and $25 \mathrm{kN} / \mathrm{m}^{2}$, $35 \mathrm{kN} / \mathrm{m}^{2}, 33 \mathrm{kN} / \mathrm{m}^{2}, 5 \mathrm{kN} / \mathrm{m}^{2}, 6 \mathrm{kN} / \mathrm{m}^{2}, 11 \mathrm{kN} / \mathrm{m}^{2}, 25 \mathrm{kN} / \mathrm{m}^{2}, 42 \mathrm{kN} / \mathrm{m}^{2}, 50 \mathrm{kN} / \mathrm{m}^{2}, 62 \mathrm{kN} / \mathrm{m}^{2}$, respectively. Cellular vortex technique with relative little mathematics has been established to have recorded successes in numerical modeling of fluid flow in aquifer simulation.
\end{abstract}

Key words: Numerical, Darcy, vorticity, image, subsurface porous media.

\section{Nomenclature}

A Crossectional area, $\mathrm{L}^{2}, \mathrm{ft}^{2}\left(\mathrm{~m}^{2}\right)$

$A_{x}, A_{y} \quad$ Crossectional area normal to $\mathrm{x}, \mathrm{y}$ direction,

$A_{x}, A_{y} \quad$ respectively, $\mathrm{L}^{2}, \mathrm{ft}^{2}\left(\mathrm{~m}^{2}\right)$

$k_{w} \quad$ Absolute permeability of water $\left(\mathrm{L}^{2}\right)$

$\mu_{w} \quad$ Viscosity of water (poise, milli-darcy, md)

Fluid volume formation water $\mathrm{L}^{3} / \mathrm{L}^{3}$,

$B_{w} \quad \mathrm{RB} / \mathrm{SCF}$ (reservoir barrels per standard cubic feet) $\left(\mathrm{m}^{3} / \mathrm{stdm}^{3}\right)$

$V_{R} \quad$ Aquifer total volume, $\mathrm{ft}^{3}\left(\mathrm{~m}^{3}\right)$

$q_{w} \quad$ Volume flow rate of water $\left(\mathrm{m}^{3} / \mathrm{s}\right)$

$\Phi_{w} \quad$ Potentials water $\left(\mathrm{m} / \mathrm{Lt}^{2}\right)$, psia $(\mathrm{kPa})$

$\phi \quad$ Porosity, fraction

$\Gamma \quad$ Circulation

$\omega \quad$ angular acceleration $(\mathrm{rad} / \mathrm{s})$

$S_{s} \quad$ Storability

G Transmissivity

Corresponding author: Oyetunde Adeoye Adeaga, Dr., research field: computational fluid dynamics.
$\begin{array}{ll}G_{0} & G / S_{s} \\ H & \text { Head drawdown (m) }\end{array}$

\section{Introduction}

A subsurface porous media is a trap in which fluids such as oil, gas and/water have accumulated over millions of years by migration from source rocks, decays of aquatic remains etc. A porous medium/material is a solid (often called frame or matrix) permeated by an interconnected network of pores (voids) filled with a fluid (liquid or gas). Usually both the solid matrix and the pore network (also known as the pore space) are assumed to be continuous, so as to form two or more interpenetrating continua as in a sponge. Many natural substances such as rocks, soils, biological issues (e.g., bones, skin) and 
man-made materials such as cements, foams and ceramics can be considered a porous media, A poro-elastic medium is characterized by its porosity, permeability as well as the properties of its constituents (solid matrix and fluid) [1].

The concept of porous media is used in many areas of applied science and engineering; mechanics, acoustics, geo-mechanics, soil-mechanics and rock-mechanics, engineering (reservoir engineering, petroleum engineering, construction engineering), geosciences (hydro-geology, petroleum geology, geophysics) biology and biophysics, material sciences etc. [2]. The numerical equations governing the flows of scientifically separable but physically immiscible fluids in subsurface earth (porous media) flow are inherently nonlinear, and the geometries and material properties characterizing many empirical problems in under-groundwater engineering can be quite irregular. More often than not, numerical simulation offers the only viable approach to the modeling of single-phase and multi-phase/multi-species flows [3].

Ewing, et al. [4] described numerical simulation as one which is widely used for predicting aquifer and reservoir behaviour and forecasting its performance. However, the mathematical model used in the simulation require the knowledge of subsurface properties. Since aquifer is relatively inaccessible for sampling, the measurable quantities at the well, provide the essential information for description [5]. Different solution techniques have been employed over the years from the one that is deterministic to the probabilistic, all in effort to provide suitable, economical solution technique to Navier-Stokes equation when applied to fluid flow and heat transfer problems. Among these methods are: random walk method, core expansion method, particle strength exchange, elliptic Gaussian blob amongst others. Not left out are the numerical methods of solution, these include: FDM (finite difference method), FEM (finite element method), Monte Carlo method and recently the hybrid Monte Carlo method as reported by
Ogundare [6]. Zheng, et al. [7] successfully applied the finite element method using Biot Poro-elastic approach to simulate $2 \mathrm{D}$ axisymmetric, $3 \mathrm{D}$ and full 3D reservoir in the subsistence of Venice in Italy [8]. Other numerical solution techniques include but not limited to: direct summation, vortex-in-cell, fast multi-pole method, species interface/mixing, and density interface/ stratification [9].

Common numerical methods that are used to simulate and eventually investigate fluid(s) flow in aquifers (either confined or unconfined) are finite element method, finite difference method, finite volume method and the combination of two or more of the earlier mentioned, requires relatively complex mathematical composition and analysis of matrix and matrices (i.e., inverses and transposes of sub matrices, factors and cofactors) occupies ample computer memory which consequently reduces the computation speed. Also computation of these methods are iterative in nature with the iteration value carefully chosen.

Vortex method has been found to have lighter mathematical expressions without necessarily involving expert analysis. It provides solutions at any location (either on-grid or off-grid) within the domain of interest unlike FEM and FDM that has rigidly grid based solutions. It is not iterative in nature, hence reduces computation time while few computer memory resource is utilized. The major importance of cellular vortex method modeling is that, each cell within the domain can be investigated in isolation or as a whole depending on the concerns of the analyst. Also the solution of each cell is as a result of the unpredictable nature of other neighbouring cells with those cells closer to the yield cell experiencing highest influence, i.e., the farther a cell to the yielding cell, the lesser the influence of yield and the aquifer rate on the particular cell.

\section{Vortex Element Method}

General vortex element method is a langragian technique for obtaining solution to engineering 
problems either in fluids or solids analysis and so does the cellular vortex method. It came to being in 1931 with the Rosenhead calculation of the Kelvin-Helmholtz instabilities. However, in 1967, Batchelor obtained solutions for the differential equation:

$$
\begin{aligned}
& \frac{\partial w}{\partial t}=v\left(\frac{\partial^{2} \omega}{\partial x^{2}}+\frac{\partial^{2} \omega}{\partial y^{2}}\right) \text { as } \\
& \omega(r, t)=\frac{\Gamma}{4 \pi v t} \exp \left(-\frac{r^{2}}{4 v t}\right)
\end{aligned}
$$

Then, Chorin applied the solution obtained by Batchelor to propose some other solution technique for the Navier-Stokes equations.

Summarily, the relative advantages of vortex methods over other traditional methods like FEM and FDM include but not limited to the following:

(1) Vortex methods are practically grid independent, thus eliminating numerous iterations associated with RANS (Reynolds averaged Navier Stokes) simulation and LES (large eddy simulation) as obtained in FEM and FDM;

(2) All problems are treated identically. No modeling or calibration inputs are required;

(3) Vortex methods are grid free solution methods for dynamic systems;

(4) These methods require few mathematics with or without expertise of the subject.

Furthermore, the vortex method is synonymous with fluid rotation and it is basically important in that, it closely study the seemly difficult fluid motion by the introduction of vortex into the porous media which principally contains rotational fluid stream within and around solid matrix.

\section{Methodology}

The control volume within the media is segregated into vertical stratums and with horizontal cell arrangements. Vortices (in case of multiple image analysis) are virtually introduced into the yielding well. The vertex/vortices displays through image(s) the intensity of vertex (mono image) or vortices (multiple images) diffusion, convection and acceleration to and fro close and distant neighbouring cells taking into account their associated viscosity, mobility, media porosity, permeability, adhesive and cohesive forces. Conventional methods for solving the Navier-Stokes equations, which govern fluid motion, typically require a computational mesh of the fluid-media domain. If the geometry of the fluid domain takes complicated shapes, mesh generation often becomes the major bottleneck in the overall simulation process. Furthermore, exterior flow problems such as the flow around an airfoil require artificial boundary conditions or other trickery. Vortex methods are mesh-free, particle based discretization which do not suffer from these disadvantages. Mesh independence in this context means that, only the boundaries of the computation domain-not the domain itself - needs to be described using a surface mesh. This greatly simplifies the meshing process and allows infinite domains [10].

\subsection{Vorticity Evolution Equations}

The complete vorticity evolution transport equation for incompressible fluids flow (presented in Cartesian coordinate), written in Laplacian form is given as [11]:

$$
\begin{aligned}
& \frac{\mathrm{D} \omega}{\mathrm{D} t}=\frac{\partial \omega}{\partial t}+\overbrace{(u \cdot \nabla) \omega}^{\mathrm{Advection}}= \\
& \underbrace{(\omega \cdot \nabla) u}_{\text {Vortexstreching }}+\underbrace{\frac{1}{\rho^{2}}(\nabla \rho \times \nabla p)}_{\text {baroclinical }} \\
& +\overbrace{\Delta \times f_{e}}^{B F}+\underbrace{\text { Viscous diffusion }}_{v \nabla^{2} \omega}
\end{aligned}
$$

The following assumptions were made in simplifying the complete vorticity evolution equation:

(1) Fluids are considered incompressible or slightly compressible;

(2) Gravitational body forces act significantly only in flow of density differences; 
(3) Pressure differences or relative movement of boundaries are caused by fluid motion/dynamics.

Therefore, a more suitable/adaptable vorticity equation is given as:

$$
\begin{aligned}
& \frac{\mathrm{D} \omega}{\mathrm{D} t}=\frac{\partial \omega}{\partial t}+\overbrace{(u \cdot \nabla) \omega}^{\text {Advection }}= \\
& \underbrace{(\omega \cdot \nabla) u}_{\text {Vortexstreching }}+\text { Viscous diffusion }
\end{aligned}
$$

\section{Vortexstreching}

Expanding the 2D equation and rearranging in Cartesian and radial coordinate, respectively, and assuming negligible effects of advection, vortex stretching, we have:

$$
\begin{gathered}
\frac{\partial \omega}{\partial t}=v\left(\frac{\partial^{2} \omega}{\partial x^{2}}+\frac{\partial^{2} \omega}{\partial y^{2}}\right) \\
+\varepsilon\left(\frac{\partial \omega}{\partial x}+\frac{\partial \omega}{\partial y}\right)+\psi\left(\frac{\partial u}{\partial x}+\frac{\partial v}{\partial y}\right) \\
\frac{\partial \omega}{\partial t}=\frac{v}{r} \frac{\partial}{\partial r}\left\{r \frac{\partial \omega}{\partial r}\right\}
\end{gathered}
$$

therefore required to express the 2-Dimensional form of Eq. (3), in the form that represents Eq. (4).

\subsection{Basic Aquifer Numerical Equations}

Assumptions made were: Laminar steady flow, constant compressibility (geometric), constant pressure i.e., at particular depth pressure is constant, time invariant constituent's mobility, non-compressible rock (i.e., $\phi \cong$ constant).

For a 1-D (x-axis flow), the derived governing equations are [12]:

$$
\begin{aligned}
& S_{S} \frac{\partial h}{\partial t}=-\nabla \cdot q-G \\
& S_{S} \frac{\partial h}{\partial t}=-\nabla \cdot(-K \nabla h)-G \\
& S_{S} \frac{\partial h}{\partial t}=K \nabla^{2} h+\nabla h-G \\
& \frac{\partial h}{\partial t}=\alpha \nabla^{2} h+\beta \nabla h-G_{0}
\end{aligned}
$$

Phase equations:

$$
A_{x} \frac{\partial}{\partial x}\left\{\frac{k_{w}}{\mu_{w} B_{w}} \frac{\partial \Phi_{w}}{\partial x}\right\}+q_{w}=V_{R} \frac{\partial}{\partial t}\left(\frac{\varphi S_{w}}{B_{w}}\right)
$$

Eq. (11) on expansion becomes:

$$
\begin{aligned}
& A_{x}\left(\frac{k_{w}}{\mu_{w}}\right)\left(\frac{B_{w}}{B_{w}}\right)\left(\frac{\partial^{2} \Phi_{w}}{\partial x^{2}}\right)+A_{x} B_{w} \frac{\partial}{\partial x}\left(\frac{k_{w}}{\mu_{w} B_{w}}\right) \frac{\partial \Phi_{w}}{\partial x}+B_{w} q_{w}=\left(\frac{B_{w}}{B_{w}}\right) V_{R} \varphi \frac{\partial S_{w}}{\partial t}+B_{w} V_{R} S_{w} \frac{\partial}{\partial t}\left(\frac{\varphi}{B_{w}}\right) \\
& \text { gives } \\
& A_{x}\left(\frac{k_{w}}{\mu_{w}}\right)\left(\frac{\partial^{2} \Phi_{w}}{\partial x^{2}}\right)+A_{x} B_{w} \frac{\partial}{\partial x}\left(\frac{k_{w}}{\mu_{w} B_{w}}\right) \frac{\partial \Phi_{w}}{\partial x}+B_{w} q_{w}=B_{w} V_{R} \frac{\partial}{\partial t}\left(\frac{\varphi S_{w}}{B_{w}}\right)
\end{aligned}
$$

Introducing Laplacian compartment to obtain the general aquifer numerical model as:

$$
\begin{aligned}
& A_{c}\left[\left(M_{w}\right)\right] \nabla^{2} P_{w}+A_{c}\left[B_{w} \nabla\left(\frac{M_{w}}{B_{w}}\right)\right] \nabla P_{w}+A_{c}(g h)\left[\left(M_{w} \nabla^{2} \rho_{w}\right)\right]-\left(B_{w} \nabla\left(\frac{M_{w}}{B_{w}}\right) \nabla P_{c w}\right)-\left[\left(M_{w}\right) \nabla^{2} P_{c w}\right]+\left(B_{w} q_{w}\right) \\
& = \\
& V_{R} \varphi\left[-\left\{\left(\frac{S_{w}}{B_{w}}\right) \frac{\partial B_{w}}{\partial P_{w}}\right\}\right] \frac{\partial P_{w}}{\partial t}
\end{aligned}
$$

Expanding Eq. (12) and dividing through by the coefficient of $\frac{\partial P_{w}}{\partial t}$ and rearranging gives the equivalent vortex expression/equation thus, Eq. (9) gives the mathematical model for, 2-Dimensional aquifer as thus. 


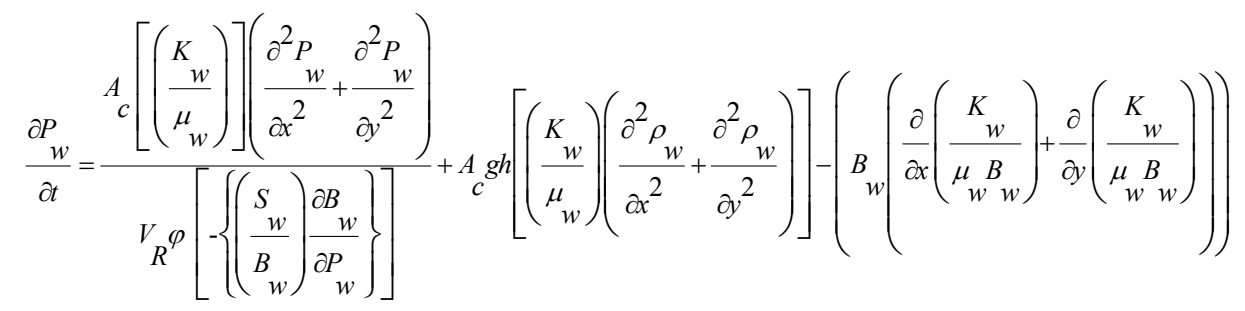

$$
\begin{aligned}
& \frac{+A_{c} g h\left[\left(\frac{K_{w}}{\mu_{w}}\right)\left(\frac{\partial^{2} \rho_{w}}{\partial x^{2}}+\frac{\partial^{2} \rho_{w}}{\partial y^{2}}\right)\right]-\left(B_{w}\left(\frac{\partial}{\partial x}\left(\frac{K_{w}}{\mu_{w} B_{w}}\right)+\frac{\partial}{\partial y}\left(\frac{K_{w}}{\mu_{w} B_{w}}\right)\right)+\left(\frac{\partial P_{c w}}{\partial x}+\frac{\partial P_{c w}}{\partial y}\right)-\left[\left(\frac{K_{w}}{\mu_{w}}\right)\left(\frac{\partial^{2} P_{c w}}{\partial x^{2}}+\frac{\partial^{2} P_{c w}}{\partial y^{2}}\right)\right]+\left(B_{w} q_{w}\right)\right.}{V_{R} \varphi\left[-\left\{\left(\frac{S_{w}}{B_{w}}\right) \frac{\partial B_{w}}{\partial P_{w}}\right\}\right]} \\
& \psi_{d} \text { (diffusive coefficient) }=\frac{A_{c}\left[\left(\frac{K_{w}}{\mu_{w}}\right)\right]}{V_{R} \varphi\left[-\left\{\left(\frac{S_{w}}{B}\right) \frac{\partial B}{B_{w}}\right)\right.} \\
& \psi_{c x} \text { (convective coefficient) }=\frac{A_{c}\left[B_{w}\left(\frac{\partial}{\partial x}\left(\frac{K_{w}}{\mu_{w}{ }_{w}}\right)\right]\right.}{V_{R} \varphi\left[-\left\{\left(\frac{S_{w}}{B}\right) \frac{\partial B}{w}\right)\right]} \\
& \psi_{c y}(\text { convective coefficient })={ }_{c}^{A_{c}}\left[B_{w}\left(\frac{\partial}{\partial y}\left(\frac{K_{w}}{\mu_{w}{ }^{B} w}\right)\right)\right] \\
& V_{R} \varphi\left[-\left\{\left(\frac{S_{w}}{B_{w}}\right) \frac{\partial B_{w}}{\partial P_{w}}\right\}\right] \\
& \psi_{a}(\text { accelerative coefficient })= \\
& A_{c} g h\left[\left(\frac{K_{w}}{\mu_{w}}\right)\left(\frac{\partial^{2} \rho_{w}}{\partial x^{2}}+\frac{\partial^{2} \rho_{w}}{\partial y^{2}}\right)\right]-\left(B_{w}\left(\frac{\partial}{\partial x}\left(\frac{K_{w}}{\mu_{w} B_{w}}\right)+\frac{\partial}{\partial y}\left(\frac{K_{w}}{\mu_{w} B_{w}}\right)\right)\right. \\
& +\frac{\left(\frac{\partial P_{c w}}{\partial x}+\frac{\partial P_{c w}}{\partial y}\right)-\left[\left(\frac{K_{w}}{\mu_{w}}\right)\left(\frac{\partial^{2} P_{c w}}{\partial x^{2}}+\frac{\partial^{2} P_{c w}}{\partial y^{2}}\right)\right]+\left(B_{w+} q_{w}\right)}{V_{R} \varphi\left[-\left\{\left(\frac{S_{w}}{B_{w}}\right) \frac{\partial B_{w}}{\partial P_{w}}\right\}\right]}
\end{aligned}
$$


The algorithm flowchart required for the cellular vortex method of solution. In the following steps, it will be adapted to the viscous term of the vorticity transport equation. Comparing and evaluating the equivalent vortex coefficients as below.

\section{Summary of Input Data}

An $11 \times 11 \times 16$ discretized grid (1,936-cells) system was used to test the developed aquifer program. The basic simulation data for the yielding well are shown below.

\section{Results and Discussion}

The coefficients $\psi_{s}$, are for 1-D and 2-D models. The fact that, the aquifer numerical equations can be rearranged to resemble Batchelor's second order partial differential equation of 1969 shows that, the exact solution obtained by him can be used to study and analyze the flow of fluid in aquifer.

Fig. 1 presents the flowchart of the entire simulation, while Fig. 2 depicts a sub-routine to evaluate the coefficients that are needed in the overall simulation.

At the yield layer, pressure changes are very negligible while the lower yield pressure remain the same. Figs. 3 and 4 show the conditions of the aquifer after two weeks and twelve months of non-stop continuous yield of $0.5 \mathrm{~m}^{3} / \mathrm{s}$. The black portions are those of yield while the conventional water blue coloured zones shows places of water fill.

It was evident in Fig. 5 that, the upper yield layer changes considerably due to compensation to the yield layer. As time passes, during simulation, the saturation of water remain constant but there is sharp drop in average aquifer pressure head and yield drawdown.

In Fig. 6, the average aquifer pressure per layer thus experienced a downward surge around the layer of yield and latter decreases with regular from the lowest layer i.e., layer 1 to the topmost layer, i.e., layer 16. It implies that, as one goes down the layer, the aquifer pressure drawdown and the system pressure increased. It is evident that, the average aquifer pressure drawdown dropped remarkably at the instance of yield commencement but due to compensation from neighbouring aquifers the drawdown is compensated for and increases with fluctuations as shown in Fig. 5, i.e., except at the yield layer when the effect of head variation is almost unfelt, the average head of the aquifer reduces up the aquifer layer.

The obtained results were validated using Fig. 7 with those results obtained by Ertkin, et al. [13]. The same situation occurs to aquifer pressure head as shown in Fig. 8.

The aquifer pressure head also decreases as yield rate continues with time. This is evident in Fig. 8. The implication is that, unless there is pressure compensation to the aquifer, the aquifer yield pressure may diminish with time.

Table 1 presents the summary of important user's inputs for the simulation. The results obtained from this work was validated by making appropriate comparison with existing work as presented in Table 2 .

The ANOVA (analysis of variance) at $p=0.05$ within aquifer layers were presented in Table 3. This eventually confirmed decreasing profile in aquifer pressure, head and drawdown with time and at different aquifer rate/yield. 


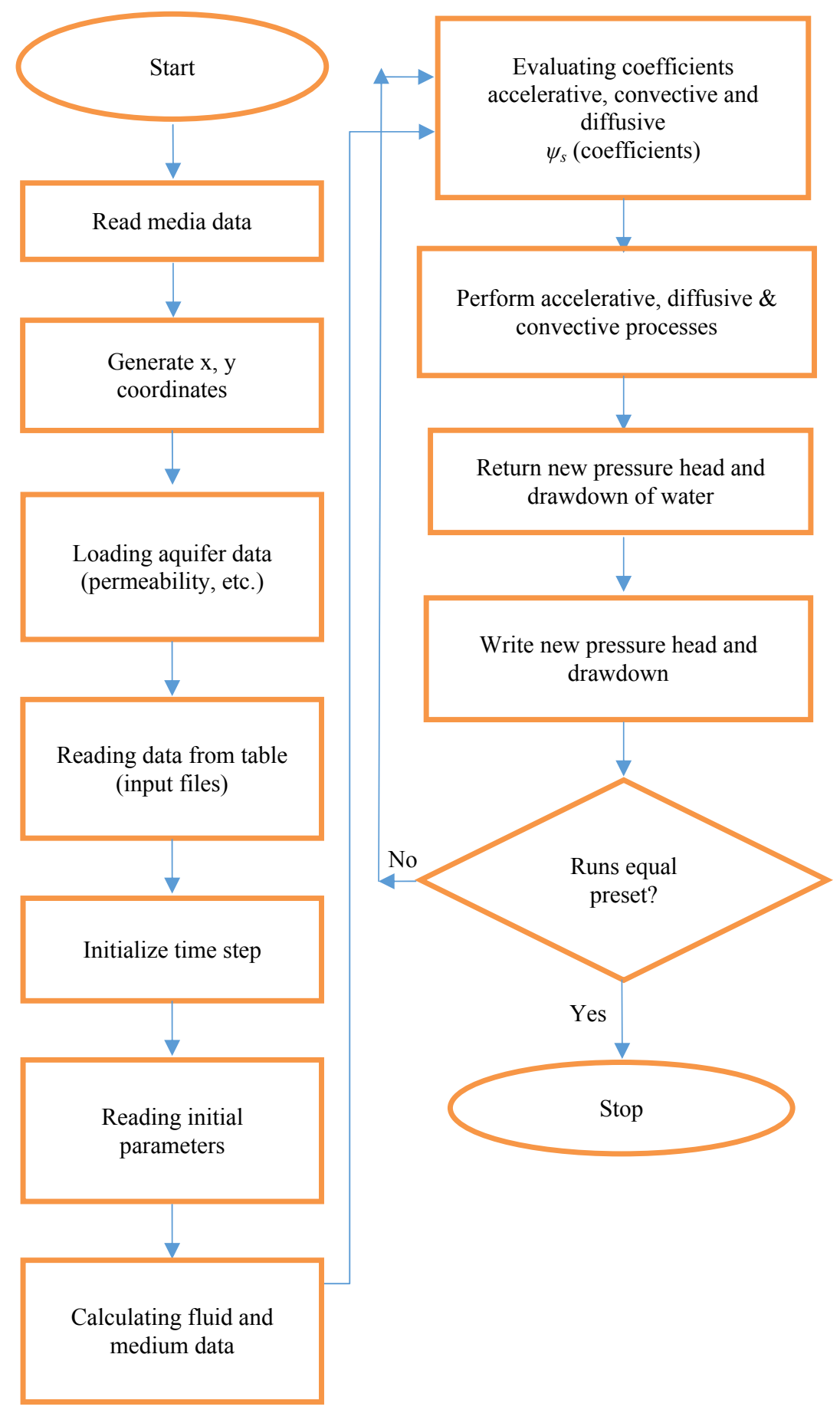

Fig. 1 Complete simulation flowchart. 


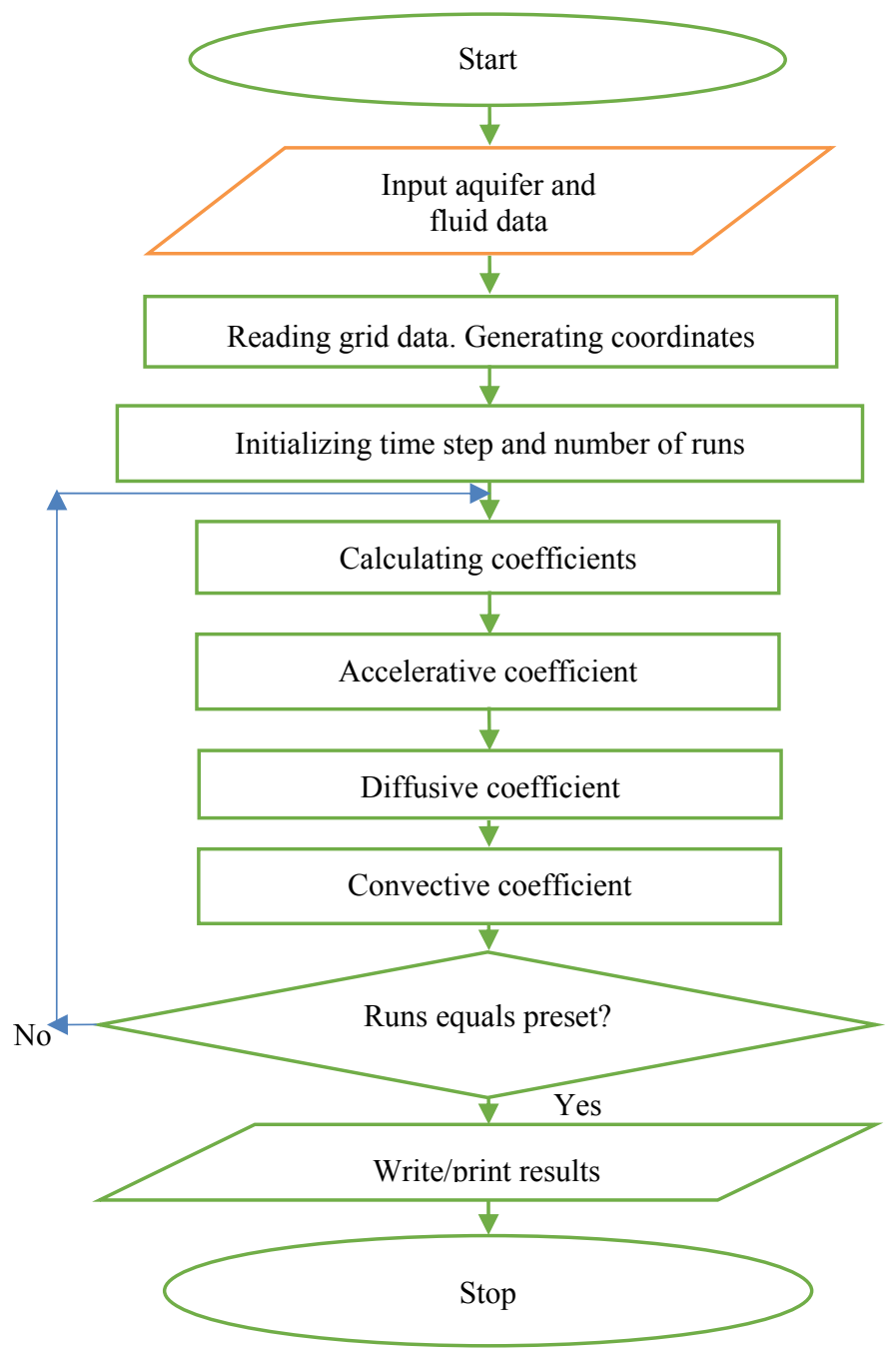

Fig. 2 Flowchart for evaluating coefficients during simulation.

Table 1 Summary of data used for simulation.

Data from the user

Well dimensions $(\mathrm{m})$

Length

Breadth

Depth

Radius of well/hole

10

10

75

Grid dimension

Divisions in x-direction 11

Divisions in y-direction

Divisions in z-direction

11

Well location $(\mathrm{m})$

$\mathrm{X}$

Y

Z

Flow rate $\left(\mathrm{m}^{3} / \mathrm{s}\right)$

\begin{tabular}{ll}
\hline Water & $0.05 \mathrm{~m}^{3} / \mathrm{s}$
\end{tabular}

Behaviour

Simulation time step (s)

Number of preset runs

86,400

Number of vortex image 


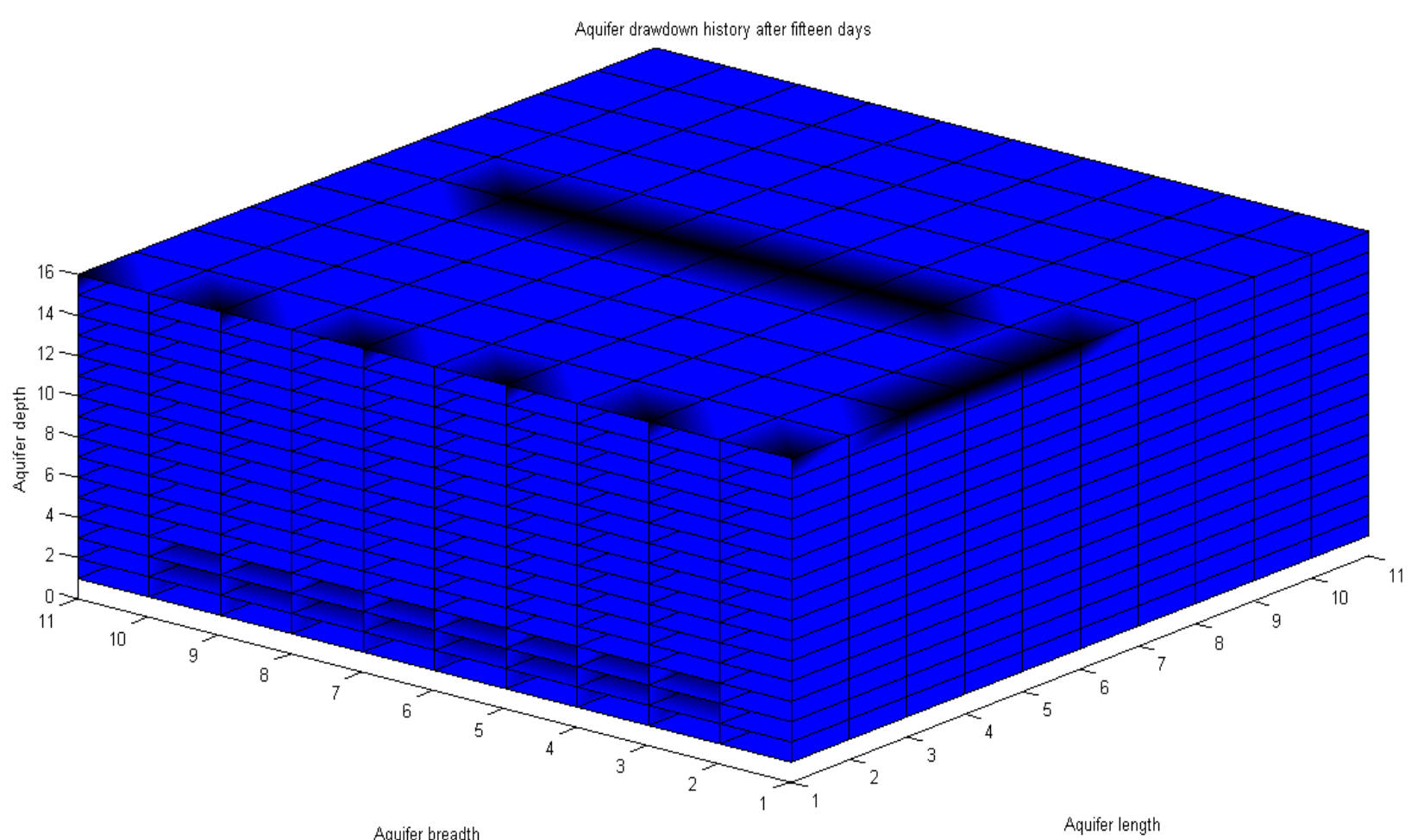

Fig. 3 3D graphical representation of aquifer condition after two months.

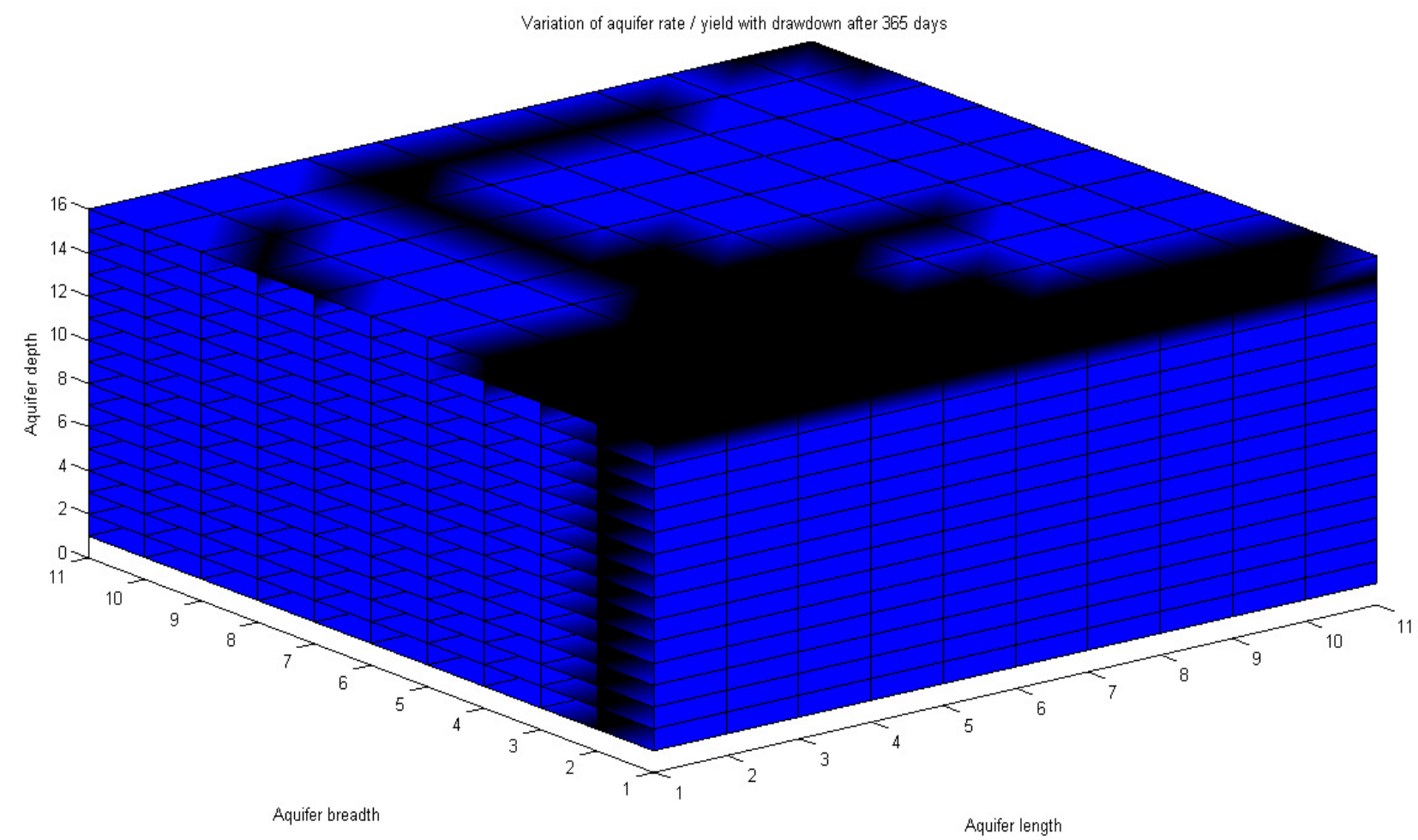

Fig. 4 3D graphical representation of aquifer condition after ten months. 


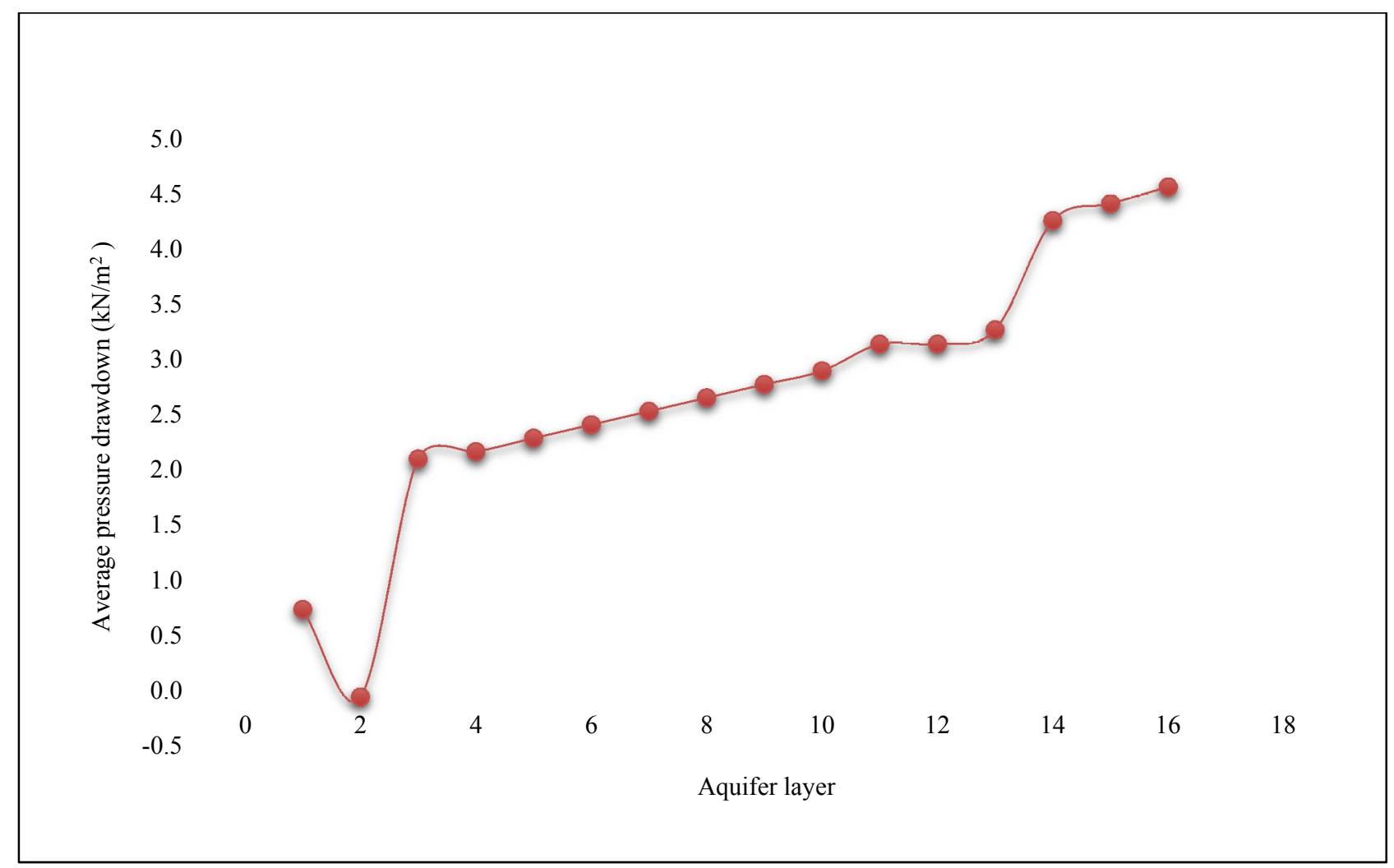

Fig. 5 Variation of aquifer average drawdown with layer.

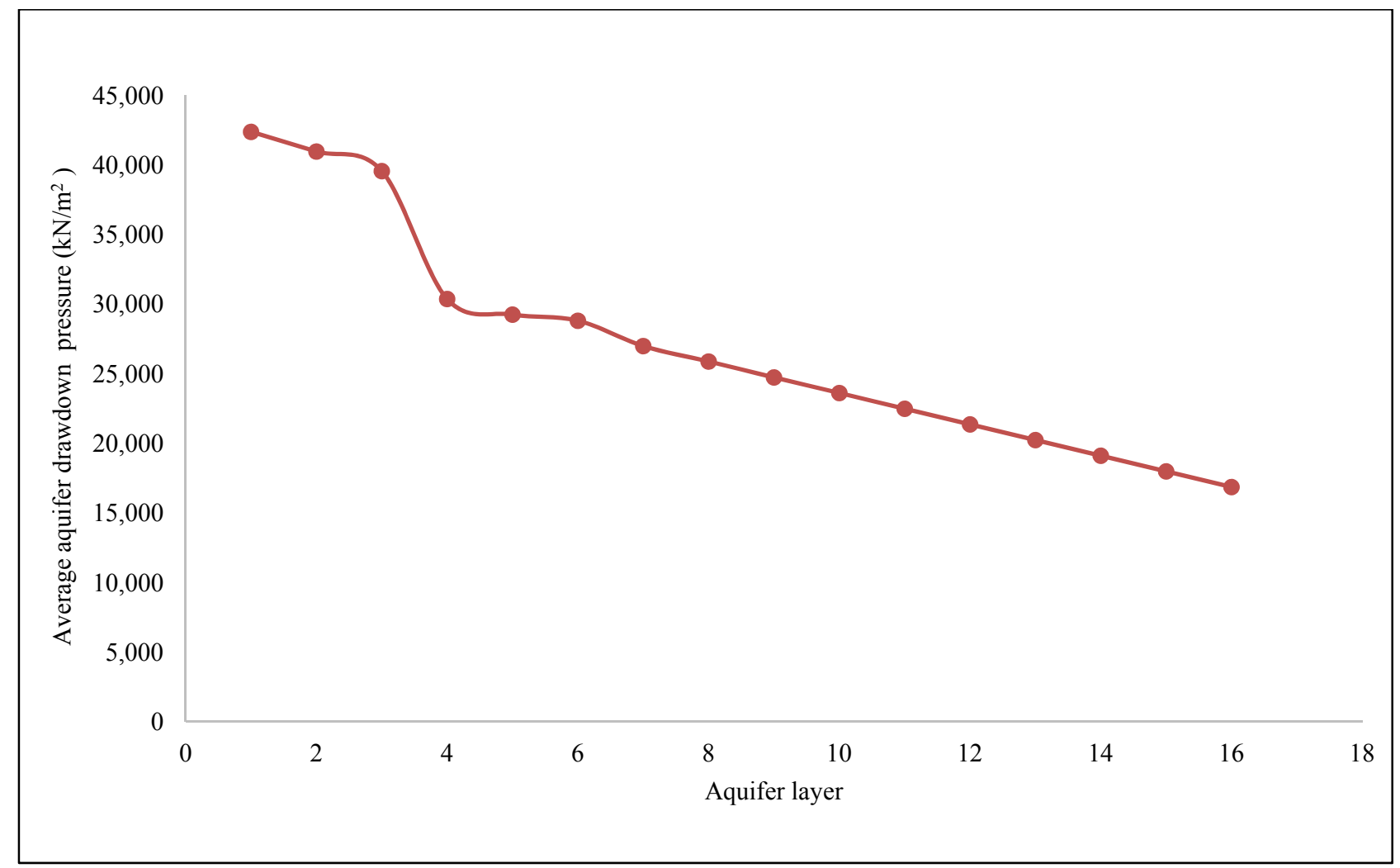

Fig. 6 Variation of average aquifer drawdown pressure with layer (Current research). 


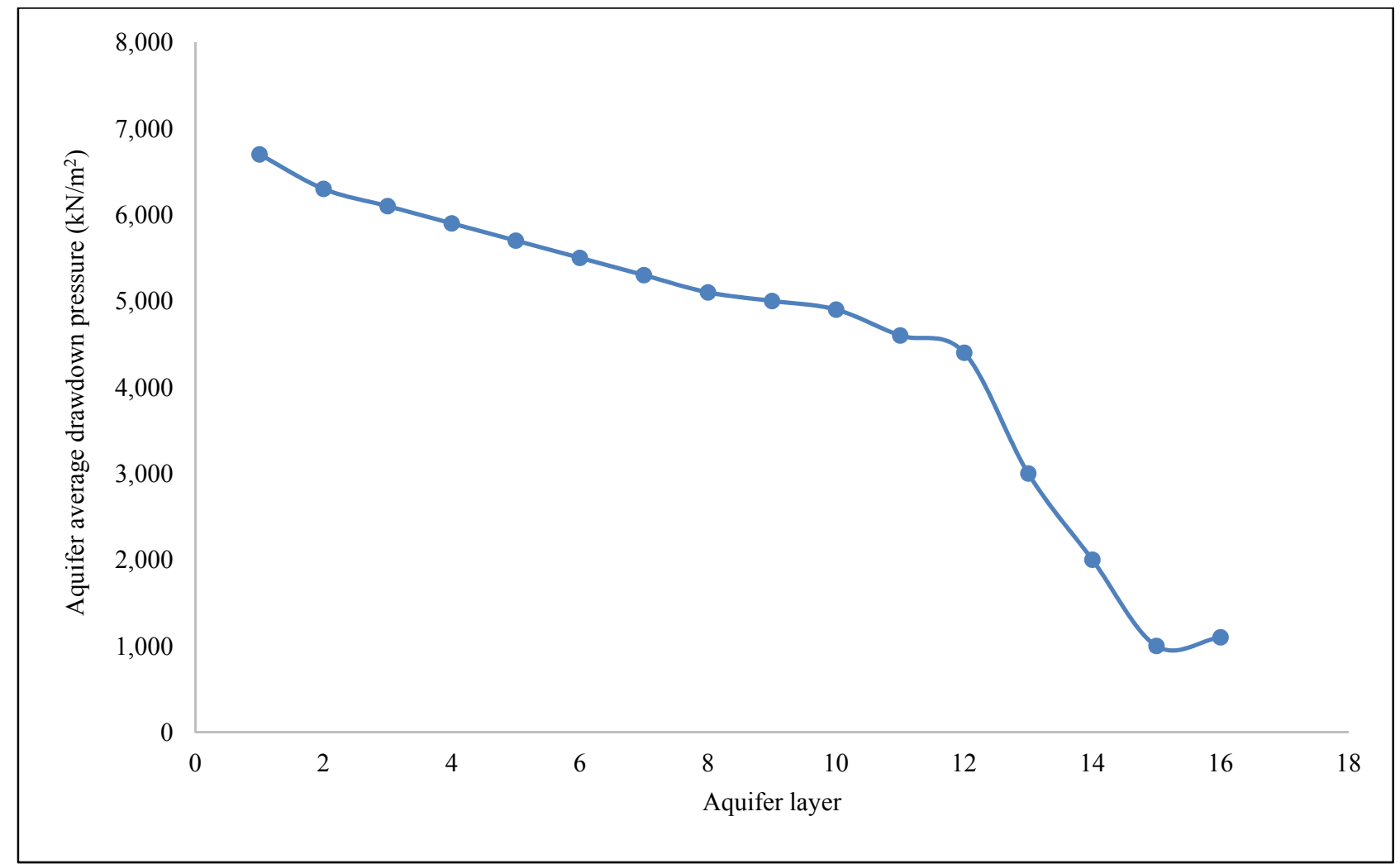

Fig. 7 Average aquifer drawdown pressure with layer. Existing work.

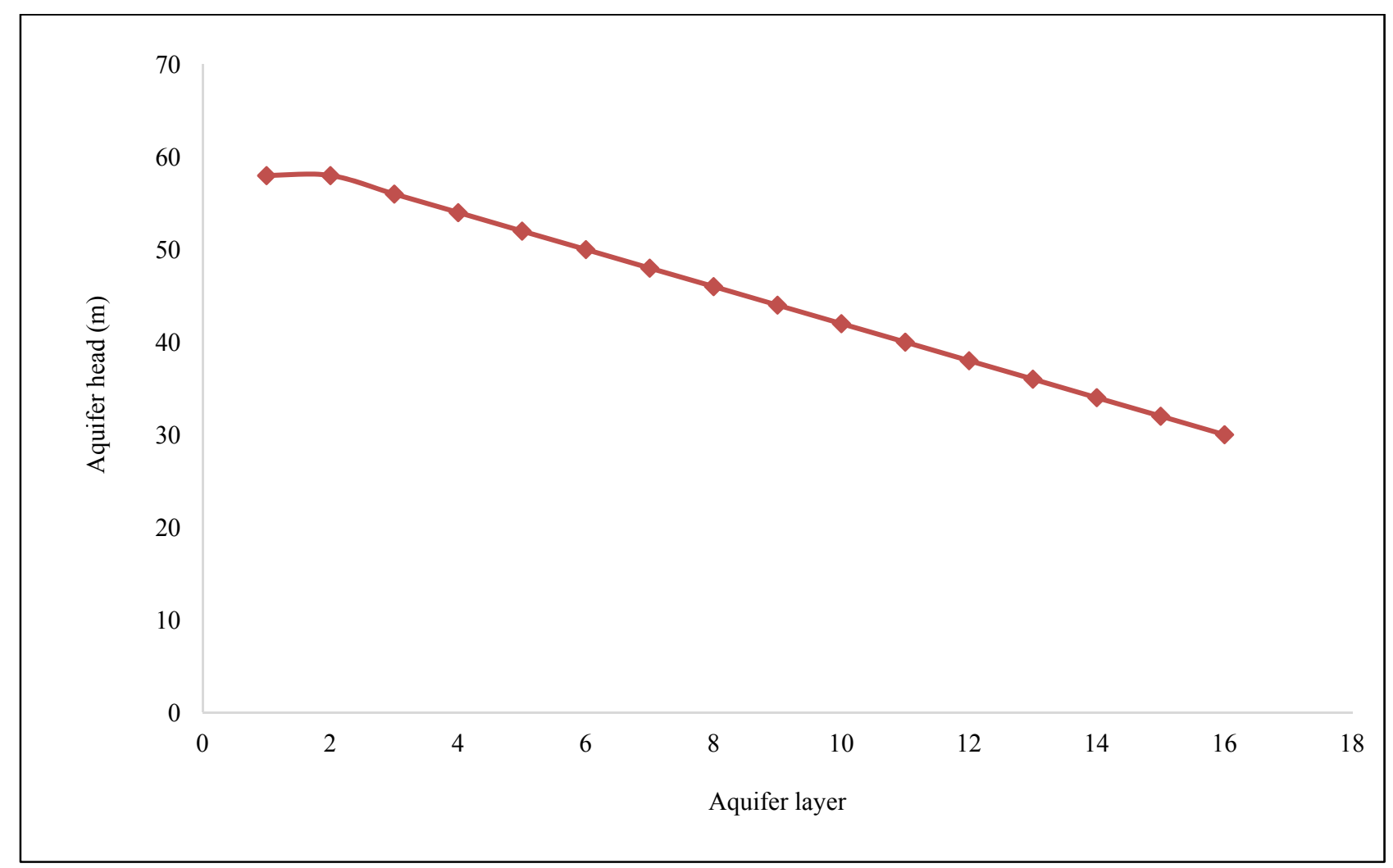

Fig. 8 Variation of average aquifer pressure head with layer. 
Table 2 Variation of different aquifer rates/yields with average drawdown pressure after 365 days.

\begin{tabular}{lllll}
\hline Aquifer rates/yield $\left(\mathrm{m}^{3} / \mathrm{s}\right)$ & Present work & Existing work & Difference & Percentage difference $(\%)$ \\
\hline 0.50 & 1.97 & 2.08 & -0.01 & -5.61 \\
1.00 & 2.06 & 2.19 & -0.03 & -6.31 \\
1.50 & 2.34 & 2.41 & -0.07 & -2.99 \\
2.00 & 2.42 & 2.52 & -0.17 & -4.13 \\
2.50 & 2.52 & 2.68 & -0.06 & -6.35 \\
\hline
\end{tabular}

Table 3 ANOVA of results of simulation.

\begin{tabular}{llllllllll}
\hline $\begin{array}{l}\text { Yield } \\
\left(\mathrm{m}^{3} / \mathrm{s}\right)\end{array}$ & $\begin{array}{l}\text { Mean } \\
\text { drawdown } \\
\left(\mathrm{N} / \mathrm{m}^{2}\right)\end{array}$ & $\begin{array}{l}\text { Mean } \\
\text { head } \\
(\mathrm{m})\end{array}$ & $\begin{array}{l}\text { Mean } \\
\text { pressure } \\
\left(\times 10^{4}\right)\end{array}$ & $\begin{array}{l}\text { Standard } \\
\text { deviation, } \\
\text { drawdown } \\
\left(\times 10^{-3}\right)\end{array}$ & $\begin{array}{l}\text { Standard } \\
\text { deviation, } \\
\text { head } \\
\left(\times 10^{-1}\right)\end{array}$ & $\begin{array}{l}\text { Standard } \\
\text { deviation, } \\
\text { pressure }\end{array}$ & $\begin{array}{l}\text { Draw down } \\
\text { variance } \\
\left(\times 10^{-5}\right)\end{array}$ & $\begin{array}{l}\text { Head } \\
\text { variance } \\
\left(\times 10^{-2}\right)\end{array}$ & $\begin{array}{l}\text { Pressure } \\
\text { variance }\end{array}$ \\
\hline 0.5 & 1.977 & 52.02 & 2.923 & \pm 9.97 & \pm 1.82 & \pm 3.42 & 9.94 & 3.31 & $1,169.71$ \\
1.0 & 2.046 & 50.01 & 2.604 & \pm 8.61 & \pm 1.61 & \pm 3.16 & 8.82 & 3.33 & $1,142.63$ \\
1.5 & 2.336 & 49.02 & 2.432 & \pm 8.30 & \pm 1.55 & \pm 2.92 & 8.32 & 3.22 & $1,141.36$ \\
2.0 & 2.504 & 46.33 & 2.236 & \pm 7.97 & \pm 1.03 & \pm 2.55 & 7.66 & 3.00 & $1,041.33$ \\
2.5 & 2.634 & 43.24 & 1.967 & \pm 7.62 & \pm 1.01 & \pm 2.37 & 7.21 & 2.91 & $1,001.32$ \\
\hline
\end{tabular}

\section{Conclusions and Recommendations}

\subsection{Conclusions}

The finite element method, finite difference method and finite volume method has successfully being used to study fluid flow in aquifer. This paper has revealed the potentials of cellular vortex element modeling technique as an efficient and viable alternative numerical tool for subsurface porous media fluid flow analysis with special attributes of solutions that are grid independent with little computer resource usage and also time conserving. The method is however, both probabilistic and deterministic in solution evaluation.

\subsection{Recommendations}

It is therefore recommended that, the 3D model be formulated and applied to investigate the media. Multiple vortex images should be also investigated with $1 \mathrm{D}, 2 \mathrm{D}$ and $3 \mathrm{D}$. It is also recommended that, these models be applied to other subsurface porous media to actually ascertain its generality and wider application. The axes used to formulate the models were considered to be rectilinear which of course is not in the actual sense. It is therefore also recommended that, the assumptions be relaxed to capture a little more of reality.

\section{References}

[1] Saman, P. G. 1992. Vortex Dynamics. Cambridge: Univ. Press.

[2] Lui, L., Feng, J., Jain-ren, F., and Ke-fa, C. 2005. "Recent Development of Vortex Methods in Incompressible Viscous Bluff Body Flows." Journal of Zheijang University of Science 6A (4): 283-8.

[3] Dyrdahl, J. 2014. "Thermal Flow in Fractured Porous Media and Operator Splitting." Master of science in mathematics thesis, Norwegian University of Science and Technology.

[4] Ewing, R. E., Russell, T. F., and Wheeler, M. F. 1984. "Convergence Analysis of an Approximation of Miscible Displacement in Porous Media by Mixed Finite Elements and a Modified Method of Characteristics." Computer Methods in Applied Mechanics and Engineering 47 (1-2): 73-92.

[5] Shen, S. F., and Lu, Z. H. 1985. "Random Walk Technique or Transient Heat Transfer from Flat Plate in Steady Forward Motion." In Numerical Methods in Heat Transfer, edited by Lewis, R. W., and Morgan, K. Chistester: John Wiley.

[6] Ogundare, M. 2006. "Vortex Element Modeling of Fluid and Heat Flow in Vertical Channels and Ducts." Ph.D. thesis, University of Ibadan.

[7] Zheng, Y., Robert, B., and Daniel, B. 2010. "Reservoir Simulation with the Finite Element Method Using Biot Poroelastic Approach.” Energy Journal 4: 16-29.

[8] Lugt, H. J. 1983. Vortex Flow in Nature and Technology. Malabar: Krieger Publishing Company. 
[9] Ajiroba, O. A. 2006. "Finite Difference Simulation of Fluid Flow in an Oil Reservoir." Unpublished B.Sc Project, University of Ibadan.

[10] Mark, J. S. 2002-2007. Summary of Vortex Methods Literature (A Living Document Rife with Opinion). UK: Cambridge University, 17-26.

[11] Speziale, C. G. 1987. "On the Advantages of the Vorticity-Velocity Formulation of the Equations of Fluid Dynamics.” J. Comput. Phys. 73 (2): 476-80.
[12] Rezael, A. A., and Donaldson, U. 1987. "A New Two-Dimensional Single Phase Water Flood Model for Micro-computers." In SPE Paper 16491. Vol. 6. Texas: Henry L. Doherty Memorial Fund of AIME, 101-15.

[13] Ertekin, T., Abou-Kassem, J. H., and King, G. R. 2001. "Basic Applied Reservoir Simulation." In Society of Petroleum Engineers Textbook Series. Vol. 10. Texas: Henry L. Doherty Memorial Fund of AIME, 64-88. 